\title{
POLYLINGUALE INTERTEXTUALITÄT UND KONSTITUIERUNG VON KULTURSPRACHEN
}

\begin{abstract}
Der Schreiber übt keine essentielle technische Aktion auf das Wort aus. Er verfügt über eine allen Schreibern gemeinsame Schreibweise, eine Art Koine, innerhalb derer man zwar Dialekte unterscheiden kann, selten aber Stile.
\end{abstract}

Roland Barthes $(1969,49 f)$

Die Auseinandersetzung mit Textsorten oder textuellen Mustern als Relata der interkulturellen Kommunikation entbehrt wie viele textlinguistische Theoriebildungen und Konzeptionen schnell der historischen Tiefenschärfe, insofern allein gegenwartsbezogene Aspekte grenzüberschreitender Kommunikation zum erkenntnisleitenden Interesse erhoben werden. Fraglos ergeben sich gerade bei solcher Ausblendung der Gewordenheit von Textmustern, ihrer kulturellen Determiniertheit über große sprachgeschichtliche Perioden hinweg, wichtige aktuelle Einsichten. Nicht zuletzt leisten textgestützte Untersuchungen mit kontrastiver Methode zur Gegenwartskommunikation in verschiedenen Kulturen linguistische Argumente a forteriori gegen stereotype Dequalifizierungen des Fremden und begegnen wirkungsvoll den Vorurteilen zur vermeintlich bewertbaren Kulturspezifik sprachlichen Verhaltens. In jüngster Zeit darf dabei die These, textuelle Muster seien als sprachliche Handlungsschemata weit weniger kultur- als vielmehr domänenbedingt, als besonders gewichtig beurteilt werden. Kann es also nicht um einen Zweifel am Wert synchron-gegenwärtiger Erörterungen und Analysen zum Textbezug der interkulturellen Kommunikation gehen, so beantworten solche Arbeiten doch nicht die sich aufdrängenden Fragen nach dem terminus post quem jeweiliger sprachkultureller Sachverhalte. Konsistente Erklärungen zum status quo der Textsorten in der interkulturellen Kommunikation benötigen folglich Bestimmungen des status quo ante. Wir präferieren also keineswegs eine Historie als kostbaren Erkenntnisüberfluss, gegen die sich bereits F. Nietzsche (1873) entschieden wandte, sondern eine geschichtliche Fokussierung der in diesem Sammelband behandelten Thematik zwecks Vollständigkeit der sachgemäßen Perspektiven. Die sprachgeschichtliche Betrachtung soll dabei auf eine grundlegende Problematik im Zusammenhang der Verbindung von Textsorten und interkultureller Kommunikation beschränkt werden, auf den Konnex von Textmusterkonstituierung und interkultureller bzw. polylingualer Kommunikation. 
Anders ausgedrückt geht es im Weiteren um die Frage nach der Bedeutung von Polylingualität und Textsorten bei der Ausprägung kultursprachlicher Strata.

Damit sind wir sogleich in der Notwendigkeit, den polysemen wenn nicht sogar vagen Ausdruck der Kultursprache näher zu definieren. Denn es liegt auf der Hand, dass Kultursprache ex negativo durchaus als ein provozierender Begriff eingesetzt werden könnte, dem dann eine implizite Abwertung nicht-kultureller Sprachen inhärent wäre. Doch Kultursprache soll in unserem Zusammenhang nicht als ein solcher Wertbegriff verstanden werden, sondern als Terminus zur Bezeichnung von Sprachen, die ein konkretes Bündel von kommunikativen Merkmalen ausgeprägt haben; sie sind damit Zweck der intrakulturellen Kommunikation, universell einsetzbar und stellen eine Bedingung der Möglichkeit interkultureller Kommunikation dar. Eine Kultursprache im hier gemeinten Sinn zeichnet sich durch weitgehende Etablierung der Merkmale Literalität, Überregionalität, Polyfunktionalität, Literarizität, Intersozialität und Philologität aus. ${ }^{1}$ Als besonders gewichtige kultursprachliche Konstituente ist dabei die Polyfunktionalität einer Sprache anzusehen. Hierunter ist die kommunikative Leistungsfähigkeit in unterschiedlichsten Domänen der gesellschaftlichen Organisation $\mathrm{zu}$ verstehen. Eine polyfunktionale Sprache ist als kommunikatives Medium in diversen Domänen geeignet und kann folglich unterschiedlichste Funktionen erfüllen. Auf die besondere Bedeutung der Polyfunktionalisierung einer Sprache im Verlauf ihrer Etablierung als Kultursprache bin ich bereits in I. Warnke (1995) eingegangen. Im Zusammenhang der interkulturellen Perspektiven soll die dort allein nationalsprachliche Untersuchung des Deutschen erweitert werden. Dabei wird weiterhin davon ausgegangen, dass die Polyfunktionalisierung einer Sprache immer als Folge der Ausweitung von Handlungsabsichten zu beurteilen, also ein Resultat pragmatischer Intentionen ist. Sprachlich werden solche Handlungsabsichten immer über die Ebene des Textes eingelöst, jedenfalls unter der Voraussetzung, dass Texte bzw. Textmuster "konventionalisierte Mittel des Sprachhandelns zur standardmäßigen Lösung gesellschaftlicher Probleme" (B. Sandig 1996, 359) darstellen.

Die textgestützte Polyfunktionalität einer Sprache resultiert nun nicht allein aus der bloßen Okkurenz von singulären Texten in unterschiedlichsten Domänen der communicatio. Jede historische und auch gegenwartsbezogene Analyse lässt schnell erkennen, dass die Funktionalität einer Sprache nicht vermittels von Textunikaten erfassbar oder gar systematisch beschreibbar ist. Vielmehr zeigt die empirische Prüfung, dass die Vernetzung der Textokkurenzen, also der Grad ihrer Komplexität ein Movens bzw. eine Variable der polyfunktionalen Geltung einer Sprache ist. Dabei gehe ich soweit, die Vernetzung von Texten untereinander sowie ihre domänenspezifischen Referenzen aufeinander als diachrone Universalie der Ausprägung von Polyfunktionalität zu bestimmen. Es wird kaum eines besonderen Hinweises bedürfen, dass hiermit der m. E. oft auch überstrapazierte Begriff der Intertextualität in die 
Modellierung der Kultursprachenetablierung eingebracht ist. Wenn auch Intertextualität unter anderem ein weitgehend literaturwissenschaftlich reservierter Modebegriff des Dekonstruktivismus ist, kann dies jedoch nicht darüber hinwegtäuschen, dass die damit bezeichnete Konnexion von singulären Textvorkommen eine auch sprachgeschichtlich höchst bedeutsame Ebene der Diskursorganisation bestimmt. Julia Kristevas $(1969,146)$ These von der absoluten Intertextualität setzen wir daher als Axiom der weiteren Überlegungen ein: "(...) tout texte se construit comme mosaïque de citations, tout texte est absorption et transformation d'un autre texte." Dieselbe folgenreiche These hat unter anderen auch V. Leitch $(1983,59)$ nochmals in die Diskussion eingebracht, er schreibt: "The text is not an autonomous or unified object, but a set of relations with other texts". Vor diesem theoretischen Hintergrund erklärt sich Polyfunktionalisierung folglich als fortschreitender Prozess der intertextuellen Referentialität, der Knüpfung eines textuellen Netzes. Dieses erst weist den Einzeltexten je spezifische Positionen in der Organisation eines Diskurses zu, und die Diskurse wiederum sind maßgebliche Felder der sprachgeschichtlich wirksamen Kommunikation. Denn wie der Einzeltext kaum geeignet ist, textmusterbezogenen Sprachwandel zu motivieren, so kann eine diachrone Dynamik nachdrücklich erst erfolgen, wenn Texte Bezüge auf die bereits gesetzten kommunikativen Inhalte vornehmen. Der Begriff der Intertextualität supponiert nun zumeist eine Beschränkung des analytischen Zugriffs auf Vernetzungen von Texten mit sprachlicher Identität. Für die Beschreibung oder Untersuchung von Phasen einer bereits ausgeprägten nationalsprachlichen Autonomie mag dies auch noch cum grano salis legitim sein, für Perioden jedoch, die in hohem Maße durch interkulturellen Kontakt bzw. durch praktizierte Mehrsprachigkeit geprägt sind, darf eine nationalsprachlich begrenzte Bestimmung von Intertextualität kaum als angemessen gelten. Ein monolingualer Intertextualitätsbegriff nimmt Einschränkungen des Untersuchungsgegenstandes vor, die geschichtlichen Epochen ohne sprachlich definierte nationale Identität und autarke Texttraditionen nicht gerecht werden. Zeigt sich dies greifbar in den gegenwärtigen Tendenzen zur Internationalisierung von Kommunikation und der damit einhergehenden Auflösung von nationalsprachlich gekennzeichneten Textmustern, so ergeben sich im Blick auf Phasen der Konstituierung von Kultursprachen im definierten Sinn similäre Verhältnisse.

Vor einer polyfunktionalen Geltung des Deutschen steht bekanntlich eine ausgeprägte Latinität, denn das Latein war als lingua franca ein bis zum Beginn des Spätmittelalters in der öffentlichen Kommunikation des deutschen Sprachraums kaum in Frage gestelltes Primärmedium der Vertextung. Die Untersuchung der Substituierung mittelalterlicher Latinität durch volkssprachige Vertextungen führt unter der Voraussetzung der Annahme einer intertextuellen Vernetzung als Bedingung der Möglichkeit von Polyfunktionalität zwangsläufig zur Analyse von Textreferenzen, die über die Grenzen nationalsprachlicher Geltungsradien hinausgehen. In concreto ist damit eine Behandlung des Texttransfers insbesondere von lateinisch vorgeprägten Textmustern $\mathrm{zu}$ volkssprachigen Textualisierungen $\mathrm{zu}$ verstehen. Die weiteren konzeptionellen und methodologischen Implikationen der geschichtlichen Behandlung 
der interkulturellen Aspekte von Textmustern sollen hier am Beispiel der juridischen Diskursdomäne behandelt werden. Die Konzentration gerade auf diesen Ausschnitt öffentlicher Kommunikation begründet sich aus dem wichtigen Beitrag, den die Rechtsvertextungen an der Ausprägung volkssprachiger Texttraditionen in allen europäischen Kultursprachen geleistet haben. Im Segment des juridischen Diskurses entstehen in ganz Europa frühe volkssprachige Prosatexte, die damit nicht nur maßgeblich zur Etablierung volkssprachiger Verständigungsformen beigetragen, sondern zudem wirksame Größen bei der Substituierung lateinischer Vertextungstraditionen dargestellt haben.

Insbesondere die überlieferten Quellen des juridischen Diskurses zeigen, dass weder für das Spätmittelalter noch für die Frühe Neuzeit von einer autonom volkssprachigen Texttradition ausgegangen werden kann. Sowohl unter dem Gesichtspunkt der Filiation frühester volkssprachiger Vertextungen als auch für die Tradierung bereits früh etablierter Textmuster kommt dem Latein eine unbezweifelbar wichtige Bedeutung zu. Die Frage nach dem Grad der intertextuellen Relationierung weist mithin eine Verbindung zur allgemeinen Einordnung des Lateins innerhalb der deutschen Sprachgeschichtsschreibung auf, wobei in unserem Zusammenhang allein der Konnex mit dem interkulturellen Movens der Textmustergenesen interessant ist. Wird dem Latein in der neueren Forschungsliteratur ${ }^{2}$ auch nicht der Rang einer überdachenden Kultursprache des Mittelalters abgesprochen, so herrscht doch Uneinigkeit darüber, ob die abnehmende Bedeutung des Lateins in einen kontinuierlich zu modellierenden Prozess der Wandlung des Deutschen vom Althochdeutschen bis zur Gegenwart als ein Teilaspekt einzupassen ist, folglich als koordinierter Faktor neben anderen Vereinheitlichtungsursachen $\mathrm{zu}$ gelten hat, oder ob nicht vielmehr die volkssprachige Prosaokkupation der zuvor lateinisch besetzten Kommunikationsdomänen als einer d e r Brennpunkte in der Geschichte der Etablierung einer ausgebildeten deutschen Kulturprache anzusehen ist. Die Gewichtung der Substitution des Lateinischen über volkssprachige Mittel differiert also bei übereinstimmender Annahme des generellen Einflusses der Latinität. Gerade deshalb ordnet sich das sprachgeschichtliche Interesse an volkssprachigen Vertextungen des juridischen Diskurses den funktionsbezogenen Desideraten der Historiolinguistik in besonderem Maße zu. Die Vertextungsgeschichte von normierenden Quellen seit dem Spätmittelalter ist ohne Berücksichtigung der lateinischen Prädeterminanten nicht angemessen zu beschreiben, so dass die entsprechenden polylingualen Intertextualisierungen als strukturelles Charakteristikum des juridischen Diskurses zu bestimmen sind. Nicht zuletzt wegen der bis in das 18. Jahrhundert bei einer Vielzahl der gebildeten Schreiber/Sprecher vorauszusetzenden deutsch-lateinischen Bilingualität ist das Latein als "Muttersprache Europas" (C. Vossen 1979) in der Rechtskommunikation des deutschen Sprachraums noch lange Zeit präsent. Diese Tatsache bedeutet jedoch

2 Einen nach wie vor ausgezeichneten Überblick zu den wichtigsten Forschungen gibt die Publikation der Vorträge des Regensburger Kolloquiums "Latein und Volkssprache im deutschen Mittelalter 1100-1500" (N. Henkel/F. Palmer [Hg.] 1992). 
nicht, dass sich bis in das 18. Jahrhundert das Lateinische als Primärmedium juridischer Vertextung gehalten hat. Diese perpetuierte Fehleinschätzung, die selbst in neueren Sprachgeschichten des Deutschen noch zum Ausdruck kommt, ${ }^{3}$ wird durch die Quellenlage falsifiziert, nehmen doch die deutschen Texte im Gesamtkorpus juridischer Vertextungen einen breiten Raum seit dem 13. Jahrhundert ein. Dass dieser offensichtliche Befund bisher nicht hinreichenden Eingang in die Gesamtdarstellungen zur Geschichte der deutschen Sprache gefunden hat, resultiert unter anderem aus der fehlenden oder nur marginal ausgeprägten funktionsgeschichtlichen Auseinandersetzung mit den domänenspezifischen Geltungsgraden der Latinität im deutschen Sprachraum bzw. einer Beschreibung der Ablösung der lateinischen communicatio durch volkssprachige Kodifikation. Die weit überwiegende Mehrzahl der sprachwissenschaftlichen Publikationen zum historischen Verhältnis von Deutsch und Latein setzt sich mit sprachstrukturellen Analogien, Divergenzen oder ganz allgemein mit den Auswirkungen des Sprachkontaktes beider Sprachen auseinander, wobei zudem noch eine Konzentration auf lexikalische Aspekte unübersehbar ist. Ohne lexikalische Interferenzen in Frage stellen zu wollen, erhebt sich die Frage, ob die für Sprachwandlungsvorgänge des Deutschen maßgeblichen Einflüsse des Deutschen tatsächlich grosso modo auf den Wortschatz beschränkt sind oder ob nicht vielmehr die Substituierung des Lateins als Superstratum zumindest noch des Hochmittelalters durch das Deutsche ein funktionsgeschichtlich wesentlicher Vorgang ist, den es domänenspezifisch zu beschreiben und in den Kontext interkultureller Dimensionen zu rücken gilt. Bereits $H$. Munske $(1982,238)$ hat bezüglich der Rolle des Lateins als Superstratum im Deutschen und in anderen germanischen Sprachen als Gegenstand der Sprachgeschichtsschreibung neben dem Wandel des Sprachsystems auch die bedingenden Faktoren desselben als "Geschichte und Wandel des Sprachverkehrs in einem bestimmten Sprachraum" angeführt. Trifft zudem N. Palmers $(1984,579)$ Feststellung zu, dass es vielen Bereichen der Übersetzungsliteratur gelang, "ihre lateinische Vergangenheit abzuschütteln", so kann daraus nur die Forderung resultieren, die Emanzipation des Deutschen als Kultursprache nicht nur vor dem Hintergrund der sprachstrukturellen Prägungen durch das Latein einzuordnen, sondern überdies auch als Faktor der Konstituierung eigener volkssprachiger Vertextungsverfahren überhaupt.

3 Vgl. etwa C. Wells (1990, 330): "Fachbücher, besonders juristische, blieben noch geraume Zeit in lat. Sprache; bis zur Mitte des 18. Jahrhunderts hatten die deutschen Publikationen die lateinischen in Naturwissenschaften, Philosophie und Medizin jedoch überholt." Abgesehen davon, dass bereits $\mathrm{zu}$ Beginn des 16. Jahrhunderts populär adressierte Fachliteratur zur Vermittlung des römischen Rechts in deutscher Sprache publiziert wurden und insofern eine Relativierung der Aussage sachgemäß ist, hat $C$. Wells für das Fachschrifttum durchaus Recht. Doch suggeriert seine Ausführung bei fehlenden Angaben zum sonstigen juridischen Sprachgebrauch eine Dominanz des Lateinischen bis zur Mitte des 18. Jahrhunderts in Rechtstexten überhaupt. Dies trifft nicht im mindesten zu. Im Gegenteil ist die juridische Diskursdomäne eine der ersten, die zur Entfaltung volkssprachiger Vertextungen beitragen hat, während Naturwissenschaft, Philosophie und Medizin eine späte Substituierung lateinischer Vertextungstraditionen vornehmen. 
Nun haben wir es im Hinblick auf die hier zu erörternde Verbindung von polylingualen Aspekten des Sprachwandels einerseits mit Fakten des interkulturellen Kontaktes und andererseits beim Lateinischen mit einer besonderen Größe zu tun. Das Latein repräsentiert nicht eine der deutschen Kultur grundsätzlich gegenüber zu stellende Tradition, sondern ist fraglos in nachantiker Zeit ein Träger der europäischen Kulturgeschichte des Mittelalters insgesamt. Lateinische Prätextualisierungen können daher in Bezug auf deutsche Texte ein Relatum interkultureller Kontakte darstellen, möglicherweise handelt es sich jedoch auch lediglich um Quellen der deutschen Latinität, womit eine interkulturelle Dimension derartiger polylingualer Intertextualität nicht mehr gegeben ist.

Weniger prototypisch für die Prätextualisierungen und Testmustertransfers im juridischen Diskurs des deutschen Mittelalters und der Frühen Neuzeit, dafür jedoch in jedem Fall interkulturelle Dimensionen der Vertextungsgeschichte betreffend, sind solche hypertextuellen Relationen, die über die Grenzen volkssprachiger Strata im eigentlichen Sinn hinausgehen. Wenn auch das Latein als Sprache der meisten Prätexte in diversen kommunikativen Domänen in Betracht kommt, kommt eine um Vollständigkeit der Beschreibung bemühte Darstellung texthistorischer Tendenzen nicht umhin, Prätextualisierungen und Texttradierungen anderer europäischer Sprachen zu berücksichtigen. Und dies besonders, wenn es um die Erfassung von polylingualer Intertextualität als Bedingung des Ausbaus einer polyfunktionalen Leistungsfähigkeit von Volkssprachen ebenso geht wie um die Interpolation auf interkulturelle Faktoren des Sprachwandels. Ferner ist die deutsch-lateinische Intertextualität selbst noch nach der Tradierungsrichtung zu differenzieren. Es ist keineswegs unwesentlich, ob ein Text ziel- oder ausgangssprachig mit dem Latein gekoppelt ist. In der Regel ergeben sich für den juridischen Diskurs intertextuelleVerschränkungen in der Richtung vom Lateinischen zum Deutschen. Die zahlreichen Textüberlieferungen dieses Typs entsprechen dabei der Einordnung deutscher Textgeschichte als Ablösung vom Latein bzw. dessen Substituierung. Wenn auch nicht mit dieser Auftretenshäufigkeit belegt, so doch sprachgeschichtlich ebenso wichtig, weil kulturgeschichtlich bedingt, sind die Textverschränkungen der Richtung vom deutschen Ausgangstext zum lateinischen Zieltext. So wurden etwa die Landläufigen Kulmischen Rechte in ihrer verbesserten und überarbeiteten Version aus dem Jahr 1553 zwecks besserer Verständlichkeit für die polnischen Textadressaten als Jus Culmense emendatum von Caspar Schütz, der sich auch als Casparus Schutzius bezeichnete, in das Lateinische übersetzt; im 17. Jahrhundert folgt dann eine Übersetzung in das Polnische von Paul Kuszewicz. Ähnlich motiviert ist die lateinische Übersetzung des Landrechts des Herzogtums Preußen aus dem Jahr 1620, das als Jus Provinciale Ducatus Prussiae in Rostock 1623 gedruckt wurde. Ebensowenig wie also die polylinguale Intertextualität als Faktor der Konstituierung deutscher Kultursprachigkeit auf e i n e Transferrichtung zwischen Deutsch und Latein zu beschränken ist, sollten die Bezüge zwischen deutschen Vertextungen und Quellen anderer europäischer Sprachen bei einer Perspektive auf interkulturelle Aspekte des Sprachwandels ausgeblendet werden. Bei diesem Intertextualitätstyp ist das Deutsche jedoch weitgehend Ausgangssprache. So wurde 
das Magdeburger Weichbild aus dem letzten Drittel des 13. Jahrhunderts nicht nur in das Lateinische, sondern im 14. Jahrhundert zudem ins Tschechische und Polnische übersetzt. Ein weiteres Beispiel für derartige Tradierungen sind die Übersetzungen der spätmittelalterlichen Rechtsbücher in osteuropäische Sprachen. Dabei ist der Transfer vom Deutschen zu anderen, insbesondere osteuropäischen Sprachen ein zuverlässiger Indikator für die geistes-, kultur- und rechtsgeschichtliche Bedeutung eines Textes in Bezug auf seinen Verbreitungsradius und seine Rezeption.

Wir halten folglich für die Etablierung deutscher Polyfunktionalität drei Typen der polylingualen Intertextualität fest: Den Standardfall des Transfers vom Lateinischen zum Deutschen, dieser Umkehrung die Tradierungsrichtung, die insbesondere im ausgehenden Spätmittelalter und in der Frühen Neuzeit als Reflex auf Humanismus, gelehrtes Recht und Rezeption des römischen Rechtes belegt ist, und schließlich die intertextuelle Relationierung vom Deutschen zu anderen europäischen Sprachen.

Mit der so vorgenommenen Bestimmung von Tradierungslinien und der damit erreichten einfachen Typologie ist noch keine Aussage über die textuellen Ebenen der Intertextualität in Subdomänen des juridischen Diskurses bzw. in anderen Domänen gemacht. Hierfür gilt es, die Systemreferenz und die Einzeltextreferenz zu unterscheiden. Sind Folientexte Übersetzungen bzw. weitgehend am Ausgangstext orientierte Übertragungen, so ist fraglos von einer Einzeltextreferenz auszugehen. Dieser Referenztyp ist sprachgeschichtlich allein mit Bezug auf Bestimmungen domänenspezifischer Texttradierungen von Interesse. Ob ein überlieferter Text $x$ einem lateinischen Ausgangstext $\mathrm{y}$ oder einem anderen fremdsprachigen Zieltext $\mathrm{z}$ zuzuordnen ist, darf losgelöst von einer allgemeinen Analyse von Domänenspezifika als funktionsgeschichtlich weitgehend unwesentlich gelten. Hier setzen eher überlieferungsgeschichtliche Einzelstudien an, deren Ziel von der historischen Dimensionierung der Polyfunktionalisierung weitgehend abweicht. Funktionsgeschichtlich verwertbar ist die Kenntnis von Übersetzungen nur, wenn die jeweiligen Basis- bzw. Folientexte als Repräsentanten einer Funktionsdomäne fungieren. Es ergibt sich also ein erkennbares Primat der Bestimmung von intertextuellen Relationierungen in Teildomänen des juridischen Diskurses gegenüber der Detailanalyse von hypertextuellen Vermitttlungen singulärer Rechtsquellen. Das heißt, dass Einzeltextreferenzen lediglich dann von Bedeutung für die Aufgliederung textueller Relationen in der Funktionsdomäne des Rechts sind, wenn ihnen ein für die entsprechende Teildomäne verallgemeinerbarer Status zukommt; aufgrund der verbreiteten Homogenität juridischer Vertextungsverfahren ist dies bei der Mehrzahl der überlieferten Texte der Fall. Es folgt daraus, dass das Hauptinteresse der funktionsgeschichtlichen Historiolinguistik auf den Systemreferenzen liegt. Und um diese geht es auch, wenn wir uns fragen, welche kommunikativen Absichten zu welcher Zeit mit welchen textuellen Mitteln realisiert wurden und welchen Anteil dabei interkulturelle Determinanten des Sprachwandels haben. Dass der Textmustertransfer tatsächlich eine Voraussetzung zur Ausprägung eigener volkssprachiger Vertextungstraditionen darstellt, zeigt beispielsweise die Quellengruppe der für die spätmittel- 
alterliche Verfassungsorganisation des Deutschen Reiches bedeutsamen Reichslandfrieden, stehen diese doch in evidenter hypertextueller Verschränkung zu vorausgehenden lateinischen Landfrieden bzw. leiten sich aus den älteren Gottesfrieden ab. Die mit dem noch in deutscher und lateinischer Sprache überlieferten Mainzer Reichslandfrieden von 1235 einsetzende volkssprachige Tradition des Textmusters 'Reichslandfrieden' ist ein charakteristisches Beispiel für polylinguale Systemreferenz und damit für die Substitution des Lateins auf der Ebene textueller Muster.

Nun bleibt zu bedenken, daß die einzelnen Textmustertransfers in Abhängigkeit von ihrer Domänenzugehörigkeit und den jeweiligen Zeiträumen in unterschiedlicher Dynamik erfolgen. Dennoch ergibt sich ad summam ein klares Bild der durchgängigen Vorprägung bzw. Tradierung von textuellen Mustern im juridischen Diskurs über die Grenzen volkssprachiger Kommunikation hinaus, oder anders ausgedrückt, der textuellen Referenzen im Kontext interkultureller Kontakte. Im Segment des juridischen Diskurses zeigt sich einzig die juristische Fachliteratur bis in die Frühe Neuzeit hinein als ausgesprochen immun gegen deutsche Vertextungsabsichten; so setzte sich noch Thomas Murner (1475-1537) für seine laienadressierten juristischen Erörterungen in deutscher Sprache erheblicher Kritik durch seine Fachkollegen aus, und Ulrich Zasius (1461-1535) formuliert als einer der Hauptvertreter der humanistischen Jurisprudenz an der Schwelle zur Frühen Neuzeit ein vernichtendes Urteil über die volkssprachige Vermittlung juristischer Inhalte:

Diejenigen verdienten Züchtigung, welche jetzt die Wissenschaft des Civilrechts, die sie selbst kaum von außen kennen gelernt hätten, in die Muttersprache und allerlei Spielereien übertrugen: denn nicht genug, daß sie selber völlig unwissend seien, machten sie auch andere zu Narren. ${ }^{4}$

Der hier zum Ausdruck gebrachte Wille zum Festhalten bzw. zur Aktivierung lateinischer Schrifttraditionen in Deutschland ist im 15. und vor allem im 16. Jahrhundert eine vielfach zu beobachtende Folge des humanistischen Strebens nach einer Einheit des Geistes, die auch lingual vermittelbar sein sollte. Das gelehrte Ausbremsen von volkssprachigen Vertextungsabsichten ist jedoch wie gesagt weitgehend auf die Funktionsdomäne des juridischen Fachdiskurses beschränkt. Während das Schrifttum der gelehrten Jurisprudenz im Deutschen Reich bis in das 18. Jahrhundert lateinisch dominiert wird, erfolgt im Zuge von Aufklärung und Naturrechtslehre für die Gesetzessprache sogar ein verstärktes Bestreben nach volkssprachiger Allgemeinverständlichkeit, wie es in den großen Kodifikationen des 18. Jahrhunderts auch eingelöst wurde. Eine nicht unwesentliche Rolle spielte dabei Charles L. Montesquieus (1689-1755) Forderung nach nationaler Eigenheit jeweiligen Rechts und nach Verständlichkeit auch für die minderbegabte Bevölkerung. ${ }^{5}$

$5 \quad$ Charles de Secondat Montesquieu: De l'esprit des loix. 1748.

Seit 1748 sind deutsche Übersetzungen in mehreren Auflagen belegt, darunter:

Des Herrn von Montesquieu Werk vom Geist der Gesetze. Nach der neuesten und vermehrten Auflage aus dem Französischen übersetzt und mit vielen Anmerkungen versehen. Altenburg 1782. 
Wenngleich nicht für die hier interessierende Frage nach den interkulturellen Determinanten der polylingualen Intertextualität relevant, so sei doch vermerkt, dass die Verbreitung und der Einfluss von Montesquieus Gedanken im Europa des 18. Jahrhunderts ein weiterer Beleg für die transnationalen Dimensionen der europäischen Rechtsgeschichte ist. ${ }^{6}$ Es bleibt jedoch für die Geschichte der deutschen Rechtstexte immer zu bedenken, dass in den meisten Subdomänen die volkssprachige Vertextung bereits im 13. Jahrhundert ihren Ausgang nimmt und seit dieser Zeit eine zunehmend autonome sprachliche Geltung erhalten hat. Im Zusammenhang dieser Darstellung kann jedoch allein auf die Bedeutung der polylingualen Intertextualität im Kontext einer funktionsgeschichtlichen Historiolinguistik im Allgemeinen eingegangen und darauf verwiesen werden, dass die polylinguale Intertextualität ein wesentlicher Aspekt der Konstituierung von Kultursprachen ist. Demzufolge hat die Sprachgeschichtsschreibung ihre Perspektiven und ihr dezidiertes Forschungsinteresse auf interkulturell konstituierte Textsorten stärker als bisher zu beziehen. Wie weit die polylinguale Präfiguration und Tradierung von textuellen Mustern dabei de facto geht, soll am Beispiel des juridischen Diskurssegmentes der Seerechte gezeigt werden.

Die spätmittelalterlichen und frühneuzeitlichen Seerechte ordnen sich der Textgruppe der Sonderrechte zu, zu denen etwa auch die Deichrechte und insbesondere die zahlreichen Bergrechte gehören. Gerade das Beispiel der Sonderrechte macht deutlich, dass ein isolierter textgeschichtlicher Blick auf nationalsprachliche Okkurenzen wesentliche Traditionslinien und Herleitungen von Textmustern ausblendet. Das Seerecht ist als Handelsrecht von überregionaler und häufig auch transnationaler Bedeutung und mit den handelsbedingten internationalen Implikationen a priori wiederum in den Zusammenhang der europäischen Rechtsgeschichte gestellt. ${ }^{7}$ In allen Küstenregionen Europas waren im Spätmittelalter bereits Seerechte ausgeprägt, was auf die allseits notwendige Normierung des Seehandels bezüglich der besonderen Gefahren des Wassertransportes verweist. Von Bedeutung ist in diesem Zusammenhang bereits die antike Lex Rhodia. Dieses Seerecht der griechischen Antike erlangte unter anderem durch die subsidiäre Geltung im römischen Recht eine "universale Bedeutung" (G. Landwehr 1990, 1597) im Mittelmeerraum. Die europäischen Dimensionen der Seerechtsentwicklung gründen neben dem in einzelnen Territorien ähnlichen Normierungsbedarf dabei vor allem auf den europäischen Handelsräumen, denn der Seehandel war von vornherein national nicht begrenzt, so dass seine rechtliche Regelung bereits frühzeitig die Kontakte mit anderen Regionen einbezieht. Bereits das niederdeutsche Hamburgische Schiprecht aus dem Jahr 1299 berichtet vom Handel mit Skandinavien, Flandern, England und Frankreich, so dass G. Landwehr $(1990,1601)$ für das Spätmittelalter zusammenfassend festhält:

6 Eine ausführliche Darstellung des europäischen Rechtsverbundes gibt H. Hattenhauer (1992) in seiner Europäischen Rechtsgeschichte.

7 Eine Darstellung zur europäischen Quellenüberlieferung gibt bereits C. v. Kaltenborn (1851, 14-70). 
Für die Entwicklung des Seerechts an der Atlantik- und der Kanalküste sowie in der Nord- und der Ostsee erlangten die im 13. und 14. Jahrhundert entstandenen Rechtsgewohnheiten im Seehandel zwischen Bordeaux und Flandern sowie zwischen den Seehäfen an der Zuidersee und den Handelsorten im nördlich gelegenen Europa (in Norwegen, an der Ostsee und Hamburg) eine herausragende Bedeutung.

Bereits die sprachexternen Faktoren der Etablierung europäischer Seerechte zeigen, dass eine sprachhistorische Beschreibung der hierfür maßgeblichen Vertextungsvorgänge nicht ohne Berücksichtigung der polylingualen Vernetzung einzelner Textokkurenzen auskommt. Ein ausgezeichnetes Beispiel für die interkulturelle und interlinguale Bedingtheit konkreter Textexemplare ist das sogenannte Wysbische Seerechte, das nicht nur Ergebnis einer über 300 Jahre währenden Seegesetzgebungstätigkeit im Hanseraum ist, sondern überdies vielfältige Bezüge zu europäischen Seerechten aufweist. Die 1505 erstmals in niederdeutscher Sprache gedruckte Sammlung seerechtlicher Normen, deren Einfluss auf die Rechtspraxis der Frühen Neuzeit und auf spätere Kodifikationen sehr groß war, geht im propositionalen und textstrukturellen Kern auf die französischen Rôles ou Jugements d'Oléron zurück, die in der Mitte des 13. Jahrhunderts aufgezeichnet wurden. ${ }^{8}$ Die Rôles d'Oléron haben im Spätmittelalter aufgrund ihrer umfassenden Kompilation seerechtlicher Verordnungen eine weite Verbreitung gefunden und wurden ins Englische, Kastilische und weitere europäische Sprachen übersetzt, so im 14. Jahrhundert auch ins Flämische. Als Vonesse van Damme wurde der Text in Verbindung mit frühen Hamburger Seerechtssätzen im ausgehenden 14. Jahrhundert zur niederdeutschen Ordinancie, de de coplude unde scippers mit malcanderen holden kumuliert. In Verbindung mit lübischen Seerechtsnormen wurde dieser Text wiederum zum sogenannten Waterrecht kompiliert und erlangte in dieser Textgestalt bereits im 15. Jahrhundert im Hansegebiet normative Bedeutung. Mit der ersten Drucklegung des Textes durch Gottfried van Ghemen im Jahr 1505 in Kopenhagen setzt sich schließlich die Bezeichnung Wisbysches Seerecht durch, daneben ist die Bezeichnung Gotlansches Waterrecht gebräuchlich. Unter der Bezeichnung Wisbysches Seerecht wurde der Text "unzählige Male gedruckt, ins Hochdeutsche übertragen sowie ins Dänische, Schwedische und Englische übersetzt und von den Gerichten, teilweise bis zum Beginn des 19. Jahrhunderts, als Seegewohnheitsrecht angewandt" (G. Landwehr 1990, 1602). ${ }^{9}$ Mit Blick auf überlieferungsgeschichtliche Zusammenhänge des Textes - in unserem Verständnis also im Fokus polylingualer bzw. interkultureller Bezüge - ordnet I. Rösler (1993, 132) die Quelle als "ein Beispiel für die Weite und das Ausmaß der kulturellen Beziehungen zwischen den Seefahrt treibenden Völkern Nord- und Westeuropas" ein.

Bereits die noch vereinfachte Übersicht zur Textgenese des Wisbyschen Seerechtes zeigt uns also den hohen Grad interkultureller Vernetzungen von Textmustergenesen.

8 Zu Ursprung und textgeschichtlichen Wurzeln der Rôles d'Oléron vgl. K.-F. Krieger (1970).

9 Eine ausführliche Darstellung der Textgenese und insbesondere der Besonderheiten des Kopenhagener Druckes des Wisbyschen Seerechts gibt I. Rösler in ihrer Abhandlung zum Gotlanschen Waterrecht und seinen Beziehungen zum Kopenhagener Druck von 1505 (1993). 
Dabei können die europäischen Traditionslinien auch am Gebrauch einzelner Fachtermini abgelesen werden. So unterscheidet bereits das Hansische Seerecht von 1614 in den Abschnitten VIII und XII.2 verschiedene Formen der Haverei, also des Verlustes der Ladung oder sogar. des Schiffes während der Seefahrt. Die Begrifflichkeiten beziehen sich dabei auf fachterminologische Bedeutungsspektren, wie sie bereits Mitte des 13. Jahrhunderts in den norditalienischen Hafenstädten ausgeprägt wurden (vgl. G. Landwehr 1985, 5). Unter Berücksichtigung derart vielfältiger überstaatlicher Bezüge hält $H$. Pöhlmann $(1973,805)$ fest:

Noch stärker als beim europäischen Land-Fernhandel und seinen aus ökonomischen Zweckdienlichkeiten an internationalen Messen, Börsen- und anderen Handelsschwerpunkten erwachsenden Verkehrs- und Rechtsgemeinsamkeiten zeigt sich die verbindende Kraft des Ökonomischen beim überregionalen Seehandel.

Erstaunlich bleibt, daß angesichts einer solchen gesamteuropäischen Vernetzung der Rechtstradition das Latein als lingua franca mit seiner übereinzelsprachlichen Verständigungsfunktion nicht weit mehr Bedeutung bei der Seerechtskompilation hatte. Wir sehen mithin, dass die volkssprachige Überlieferung seit dem Spätmittelalter einen dominanten Beitrag zum Ausbau jeweiliger Seerechte leistet, wobei die polylingualen Vernetzungen unterschiedlicher Rechtstraditionen ohne das Latein auskommen, also im Zuge vieler Übersetzungs- und Übertragungsprozeduren hergestellt werden. Zu erklären ist diese komplexe Transferstruktur mit K.-F. Krieger (1987, 246), der feststellt, dass gerade das ältere Seerecht "in der Regel aus jahrzehntelanger gewohnheitsrechtlicher Übung, weitgehend unabhängig von der offiziellen staatlichen Rechtssetzung erwachsen" ist und damit nicht nur "in besonderer Nähe zur Rechtswirklichkeit, d.h. zur jeweiligen Seehandels- und Seeverkehrspraxis" steht, sondern auch zur volkssprachig-regionalen Kommunikation.

Das Beispiel der seerechtlichen Quellen respektive der Herleitung des Wisbyschen Seerechtes zeigt, wie konstitutiv polylinguale Bezüge bei der Etablierung von Textmustern sein können und wie diese kaum auf die Herleitung volkssprachiger Texte aus lateinischen Prätexten zu beschränken sind. Wobei der Texttransfer vom Lateinischen zum Deutschen durchaus auch bei den Seerechten eine Rolle spielt. So sind die ältesten Rechtsaufzeichnungen Lübecks aus dem 13. Jahrhundert, die passim auch das Schiffrecht behandeln und damit Grundlage späterer seerechtlicher Normen sind, zunächst lateinisch vertextet. Das von Albrecht von Bardewick, einem wohlhabenden Lübecker Ratsmitglied kodifizierte Lübische Schiffsrecht aus dem Jahr 1299 geht unter anderem auf eben diese frühe lateinische Stadtrechtssammlung zurück, womit die Standardtransferachse vom Lateinischen zum Deutschen zumindest noch paradigmatisch belegt ist.

Die Beipiele für polylinguale Intertextualität im Spannungsfeld von interkultureller Kommunikation und Ausbildung nationaler Textmuster sind derart zahlreich, dass die vorangehenden Konkretisierungen fraglos nur zur ersten Exemplifizierung geeignet sind. Es wird Aufgabe zukünftiger sprachgeschichtlicher bzw. funktionsgeschichtlicher Diskursanalysen sein, die interkulturellen Determinanten 
bei der Ausprägung von Diskursdomänen und ihren typischen sprachlichen Handlungsmustern empirisch im Einzelnen zu belegen und damit die These von der interkulturellen Vernetzung als Voraussetzung der Ausprägung von Intertextualität und damit wiederum Bedingung der Möglichkeit polyfunktionaler Geltung einer Sprache zu verifizieren. Damit ist Roland Barthes' $(1969,49)$ Verdikt von der Einbindung des Schreibers in eine Schreibweise oder anders ausgedrückt in eine kommunikative Domäne im Hinblick auf interkulturelle Strukturrahmen der Vertextung als sprachgeschichtliche Konzeption reformuliert.

\section{LITERATUR}

Barthes, Roland (1969): Schriftsteller und Schreiber. in: ders., Literatur oder Geschichte. Frankfurt/M., 44-53.

Hattenhauer, Hans (1992): Europäische Rechtsgeschichte. Heidelberg.

Henkel, Nikolaus / Palmer, Nigel F. [Hg.] (1992): Latein und Volkssprache im deutschen Mittelalter 1100-1500. Tübingen.

Kaltenborn, Carl von (1851): Grundsätze des praktischen Europäischen Seerechts. Bd. I. Berlin.

Krieger, Karl-Friedrich (1970): Ursprung und Wurzeln der Rôles d'Oléron. Köln/Wien. - (1987): Die Anfänge des Seerechts im Nord- und Ostseeraum (von der Spätantike bis zum Beginn des 13. Jahrhunderts). in: K. Düwel et al. [Hg.]: Untersuchungen zu Handel und Verkehr der vor- und frühgeschichtlichen Zeit in Mittel- und Nordeuropa. Teil IV. Göttingen, 246-265.

Kristeva, Julia (1969): Sémiotiké. Recherches pour une sémanalyse. Paris.

Landwehr, Götz (1985): Die Haverei in den mittelalterlichen deutschen Seerechtsquellen. Göttingen.

- (1990): Seerecht (Seehandelsrecht). in: Handwörterbuch zur deutschen Rechtsgeschichte. Bd. IV. Hg. von A. Erler und E. Kaufmann. Berlin. 1596-1614.

Leitch, Vincent B. (1983): Deconstructive Criticism. An advanced introduction. New York/London.

Munske, Horst H. (1982): Die Rolle des Lateins als Superstratum im Deutschen und in anderen germanischen Sprachen. in: P. Ureland [Hg.], Die Leistung der Strataforschung und der Kreolistik. Typologische Aspekte der Sprachkontakte. Tübingen, 237-263.

Nietzsche, Friedrich (1874): Unzeitgemässe Betrachtungen. Bd. II: Vom Nutzen und Nachtheil der Historie für das Leben. Leipzig.

Palmer, Nigel F. (1984): Zum Nebeneinander von Volkssprache und Latein in spätmittelalterlichen Texten. in: L. Grenzmann und K. Stackmann [Hg.], Literatur und Laienbildung im Spätmittelalter und in der Reformationszeit. Symposium Wolfenbüttel 1981. Stuttgart, 579-603.

Pöhlmann, Hansjörg (1973): Die Quellen des Handelsrechts. in: H. Coing [Hg.], Handbuch der Quellen und Literatur der neueren europäischen Privatrechts- 
geschichte. 1. Bd.: Mittelalter (1100-1500). Die gelehrten Rechte und die Gesetzgebung. München, 801-834.

Rösler, Irmtraud (1993): 'Dat Gotlansche Waterrecht'. Bemerkungen zu den hansischen Seerechten, insbesondere zum Kopenhagener Druck von 1505. in: H. Menke / K.-E. Schöndorf [Hg.], Niederdeutsch in Skandinavien IV. Berlin, 118-133.

Sandig, Barbara (1996): Stilwandel und ganzheitliche Analyse. in: U. Fix / G. Lerchner [Hg.], Stil und Stilwandel. Frankfurt/M. et. al., 359-394.

Stintzing, Roderich von (1880): Geschichte der Deutschen Rechtswissenschaft. Bd. I. München/Leipzig.

Vossen, Carl (1979): Latein, Muttersprache Europas. 2. Aufl. Düsseldorf.

Warnke, Ingo (1995): Typologische Aufgaben der historischen Textlinguistik. in: Linguistica XXXV/1, 95-121.

Wells, Christopher J. (1990): Deutsch: Eine Sprachgeschichte bis 1945. Tübingen.

\author{
Povzetek \\ VEČJEZIKOVNA MEDBESEDILNOST IN OBLIKOVANJE KULTURNIH JEZIKOV
}

\footnotetext{
Avtor v svojem prispevku izhaja iz jezikovno-zgodovinskega vidika, na podlagi katerega predstavi temeljno povezavo med besedilnimi vrstami in medkulturno komunikacijo. Pri tem se osredotoča na vlogo polilingvalnosti in besedilnih vrst pri oblikovanju kulturno-jezikovnih sistemov.
} 